\title{
Posttreatment Imaging in Patients with Head and Neck Cancer without Clinical Evidence of Recurrence: Should Surveillance Imaging Extend Beyond 6 Months?
}

\author{
(D) A. Gore, (D). Baugnon, (D). Beitler, (D) N.F. Saba, (D) M.R. Patel, (D) X. Wu, (D) B.J. Boyce, and (D) A.H. Aiken
}

\begin{abstract}
BACKGROUND AND PURPOSE: Early detection of residual or recurrent disease is important for effective salvage treatment in patients with head and neck cancer. Current National Comprehensive Cancer Network guidelines do not recommend standard surveillance imaging beyond 6 months unless there are worrisome signs or symptoms on clinical examination and offer vague guidelines for imaging of high-risk patients beyond that timeframe. Our goal was to evaluate the frequency of clinically occult recurrence in patients with head and neck squamous cell carcinoma with positive imaging findings (Neck Imaging Reporting and Data Systems scores of 2-4), especially after 6 months.
\end{abstract}

MATERIALS AND METHODS: This institutional review board-approved, retrospective data base search queried neck CT reports with Neck Imaging Reporting and Data Systems scores of 2-4 from June 2014 to March 2018. The electronic medical records were reviewed to determine outcomes of clinical and radiologic follow-up, including symptoms, physical examination findings, pathologic correlation, and clinical notes within 3 months of imaging.

RESULTS: A total of 255 cases, all with Neck Imaging Reporting and Data Systems scores of 2 or 3, met the inclusion criteria. Fiftynine patients (23\%) demonstrated recurrence (45 biopsy-proven, 14 based on clinical and imaging progression), and 21 patients (36\%) had clinically occult recurrence (ie, no clinical evidence of disease at the time of the imaging examination). The median overall time to radiologically detected, clinically occult recurrence was 11.4 months from treatment completion.

CONCLUSIONS: Imaging surveillance beyond the first posttreatment baseline study was critical for detecting clinically occult recurrent disease in patients with head and neck squamous cell carcinoma. More than one-third of all recurrences were seen in patients without clinical evidence of disease; and $81 \%$ of clinically occult recurrences occurred beyond 6 months.

ABBREVIATIONS: CECT = contrast-enhanced CT; HNSCCa = head and neck squamous cell carcinoma; HPV = human papillomavirus; NCCN = National Comprehensive Cancer Network; NI-RADS = Neck Imaging Reporting and Data Systems

$\mathbf{P}$ osttreatment surveillance imaging for head and neck cancer is complex and challenging. Altered anatomy from surgical resections, complex reconstructive surgery, and adjuvant radiation frequently define a new radiologic baseline. Despite these challenges, imaging surveillance plays a critical role in detecting early submucosal recurrences and is vital to optimizing salvage therapy. $^{1-3}$

Received November 4, 2019; accepted after revision April 18, 2020.

From the Departments of Radiology and Imaging Sciences (A.G., K.B., X.W., A.H.A.), Radiation Oncology (J.B.), Hematology/Oncology (N.F.S.), and Otolaryngology (N.F.S., M.R.P., B.J.B., A.H.A.), Emory University School of Medicine, Atlanta, Georgia. Paper previously presented at: Annual Meeting of the American Society of Neuroradiology, May 18-23, 2019; Boston, Massachusetts.

Please address correspondence to Ashley H. Aiken, MD, Department of Radiology and Imaging Sciences, Emory University Hospital, 1364 Clifton Rd NE, Atlanta, GA 30322; e-mail: ashley.aiken@emoryhealthcare.org

Indicates article with supplemental on-line table.

http://dx.doi.org/10.3174/ajnr.A6614
The Neck Imaging Reporting and Data System (NI-RADS) was developed to risk-stratify and standardize management recommendations on the basis of posttreatment imaging findings in patients with head and neck cancer. ${ }^{4,5}$ The NI-RADS template standardizes nomenclature and facilitates unambiguous and uniform reporting. Radiologists commit to a discrete level of suspicion that maps to a clear management recommendation to optimize patient care. The NI-RADS template includes numeric categories of $0-4$ based on index of suspicion for tumor recurrence at both the primary and nodal sites. A category of 0 reflects an incomplete examination, usually indicating a baseline examination or one without a suitable comparison. NIRADS category 1 indicates no evidence of recurrence, and these patients continue routine surveillance. Categories 2 and 3 indicate a positive imaging finding, reflecting low and high suspicion for tumor recurrence, respectively. For patients with NIRADS 2, it is recommended that clinicians consider direct visual 
inspection for mucosal abnormalities or PET imaging for deep abnormalities and subsequent follow-up with earlier imaging (typically 2-3 months instead of 6 months). Biopsy consideration is recommended for NI-RADS 3. Category 4 indicates definite recurrence, established pathologically or by definite radiologic or clinical progression. A detailed description of NIRADS has been published. ${ }^{5}$

Previous publications have established the frequency of NIRADS scores and their positive predictive value for recurrence by category. Krieger et $\mathrm{al}^{3}$ evaluated 618 targets and found $85.4 \%$ scored as NI-RADS one, 9.4\% scored as NI-RADS 2, and 5.2\% scored as NI-RADS 3, with failure rates of 3.79\%, 17.2\%, and $59.4 \%$ respectively. A recent article by $\mathrm{Hsu}$ et $\mathrm{al}^{6}$ looked only at the first posttreatment examination in 199 patients and found that the rates of treatment failure increased with each incremental NI-RADS category from 1 to $3(4.3 \%, 9.1 \%$, and $42 \%)$, with a strong association between the NI-RADS score and treatment failure (hazard ratio = 2.6 at the primary site, hazard ratio $=5.2$ in the neck).

Although many studies have demonstrated increased sensitivity for detecting disease recurrence with combined FDG-PET/CT, current evidence-based recommendations are limited in guiding imaging surveillance in these patients. ${ }^{3,7,8}$ Wangaryattawanich et $\mathrm{al}^{8}$ suggested that patients with an incomplete response (NI-RADS 2) to treatment for head and neck squamous cell carcinoma (HNSCCa) should undergo more frequent clinical and imaging surveillance compared with those with a complete response (NI-RADS 1). Imaging with PET/CT at least 12 weeks after the completion of treatment is generally agreed to be the optimal time for initial posttreatment baseline imaging, though a recent publication raises the possibility that this baseline imaging could occur as early as 8 weeks after treatment. ${ }^{7,9-12}$ However, beyond the first posttreatment examination, there remains wide variation in clinical practice, imaging surveillance technique (contrast-enhanced CT [CECT] alone, PET/CT, PET/CECT, MR imaging, and so forth), frequency, and duration.

The National Comprehensive Cancer Network (NCCN; https://www.nccn.org/professionals/physician_gls/default.aspx) is a comprehensive set of clinical practice guidelines in oncology that detail sequential management decisions and interventions and provide recommendations for clinical follow-up. These recommendations are based on knowledge of current evidencebased research and consensus-based management. Although the NCCN recommends posttreatment imaging within 6 months after treatment, concrete recommendations for a surveillance algorithm beyond this point remain ambiguous in patients with head and neck cancer due to the lack of consensus data. Currently, the guidelines state that any further re-imaging beyond 6 months can be considered on the basis of "worrisome or equivocal signs/ symptoms, smoking history, and areas inaccessible to clinical examination." A large percentage of patients with head and neck malignancies have a smoking history and are susceptible to submucosal recurrences, nearly all of which are inaccessible or difficult to discern on clinical examination. Thus, most institutions and referring providers elect to image asymptomatic patients for surveillance. Given the limited data and ambiguous guidance, it is understandable that there is wide variation in clinical practice for these patients. More concerning is that some providers may strictly adhere to the concrete recommendations from the NCCN and only image up to 6 months after treatment regardless of patient risk factors. Even after intent of curative therapy, up to $50 \%$ of patients with HNSCCa experience recurrences, most of which occur well beyond 6 months, particularly within the first 2-3 years of treatment. ${ }^{13}$ To our knowledge, there are no previous studies to document the frequency of imaging-detected recurrence in patients with asymptomatic head and neck cancer.

The purpose of our retrospective study was to evaluate the incidence of clinically occult recurrences detected by imaging alone (positive imaging findings, NI-RADS 2-4) in patients undergoing surveillance imaging at our institution. We also hypothesized that a considerable portion of clinically occult recurrences (in patients with NI-RADS 2-4) are detected beyond the NCCN recommended 6 months.

\section{MATERIALS AND METHODS}

\section{Patient Selection and Data Collection}

A Health Insurance Portability and Accountability Act-compliant, institutional review board-approved retrospective data base search of radiology reports containing the NI-RADS lexicon was performed from June 2014 to March 2018. Head and neck cancer NI-RADS reports were identified using an institutional data base search software containing a repertoire of dictated NI-RADS template reports. A search of posttreatment $\mathrm{PET} / \mathrm{CT}$ and CECT neck soft-tissue reports was conducted with queries of NI-RADS scores 2, 3, or 4 at either the primary site or the neck. The output included the total number of reports that used the NI-RADS-structured reporting system and had a suspicious finding scored with NI-RADS 2-4 in the impression section of the radiology report.

Inclusion criteria were the following: 1) patients with HNSCCa who had undergone definitive treatment; 2) patients who underwent a clinical examination by either otolaryngology, radiation oncology, or medical oncology within 3 months of imaging; and 3) continued imaging and clinical follow-up of NIRADS 2-4 lesions or biopsy of the imaging abnormality. Exclusion criteria included patients who already had a biopsyproven recurrence (local, regional, or distant) before the imaging examination.

By definition, NI-RADS 1 category denotes negative findings on an imaging examination, with "no radiologic evidence of recurrence." Therefore, any recurrence in this category would be radiographically occult. Thus, patients with NI-RADS 1 were not queried in the current study. NI-RADS 4 category represents "definitive recurrence," strictly applied at our institution to biopsyproven recurrence or clear clinical and radiographic progression, so patients are, by definition, symptomatic in the vast majority of people.

Clinical evidence of disease recurrence was defined by any clinical examination finding or symptom within the clinical note that raised concern for recurrence. Examples of suspicious clinical examination findings included new palpable abnormalities on physical examination or a finding deemed suspicious by Ear, Nose, and Throat physicians on office-based flexible fiber optic endoscopy. Common clinical symptoms related to treatment or expected sequelae of postradiation changes, including thickening 
of the skin and platysma, radiation mucositis, and thickening of the laryngeal structures/laryngeal edema, were not classified as "clinical suspicion of recurrence." Patients without worrisome symptoms or physical examination findings to indicate recurrence were deemed to have clinically occult recurrence.

\section{Image Interpretation}

All posttreatment PET/CECT and CECT scans were interpreted using the NI-RADS template by 1 of 4 head and neck-trained neuroradiologists $(31,16,11$, and 10 years of experience), whose interobserver agreement was previously shown to be $0.821 .^{3}$ For each patient, both the primary site and neck were assigned a separate NI-RADS category of either 1, 2, 3, or 4, and all NI-RADS 2 subcategories ( $2 \mathrm{a}$ and $2 \mathrm{~b}$ ) were recorded as a category 2 , because the level of suspicion is the same. Interpreting radiologists reviewed prior clinical history, physical examination, and endoscopic notes and compared baseline imaging, including pretreatment FDG avidity, when available.

\section{Surveillance Protocol}

The imaging surveillance algorithm for patients with head and neck malignancy used at our institution mirrors the algorithm published in the American College of Radiology White Paper. ${ }^{5}$ Three months following completion of definitive therapy (surgery and radiation and/or chemotherapy; chemoradiation therapy), patients undergo a whole-body PET/CT and a neck CECT. If the patient continues to demonstrate no clinical evidence of disease recurrence, he or she continues to be imaged every 6 months for 1 year with a CECT of the neck followed by another CECT of the neck 1 year thereafter. At any point during this algorithm, if there is suspicion of recurrence, the patient may undergo PET/CT imaging as well. At our institution, PET/CT imaging follows a standard protocol, and images are acquired on Discovery 600 and 690 PET/CT scanners (GE Healthcare). Patients fast for 6 hours before injection of $10-14 \mathrm{mCi}$ of FDG. For patients with a blood glucose level of $\geq 200 \mathrm{mg} / \mathrm{dL}$, imaging is deferred due to the altered biodistribution of FDG. PET/CT images are acquired from the skull vertex to the midthighs 1 hour after injection of FDG. In addition, a CECT of the neck with the arms down is performed after the PET/CT using a split-bolus technique with $55 \mathrm{~mL}$ injected at $2.5 \mathrm{~mL} / \mathrm{s}$, followed by a 40 -second delay and another $55 \mathrm{~mL}$ at the same rate, with a total scan delay of 90 seconds. Axial images are acquired from the frontal sinuses to the mediastinum at $1.25-\mathrm{mm}$ section thickness (pitch, 0.984:1; gantry rotation, 0.7 seconds; FOV, $25 \mathrm{~cm} ; 120 \mathrm{kV}$ [peak]; and smart milliampere with a noise index of 13.78). Reformatted images are reconstructed at $2.5-\mathrm{mm}$ thickness in the axial planes and $3 \mathrm{~mm}$ in the sagittal and coronal planes.

\section{Statistics}

Descriptive statistics were performed to assess the frequency of clinically occult HNSCCa recurrences in patients with NI-RADS scores of 2 or 3 .

\section{RESULTS}

Our data base search yielded a total of 404 unique cases. One hundred forty-nine of these cases were excluded because they
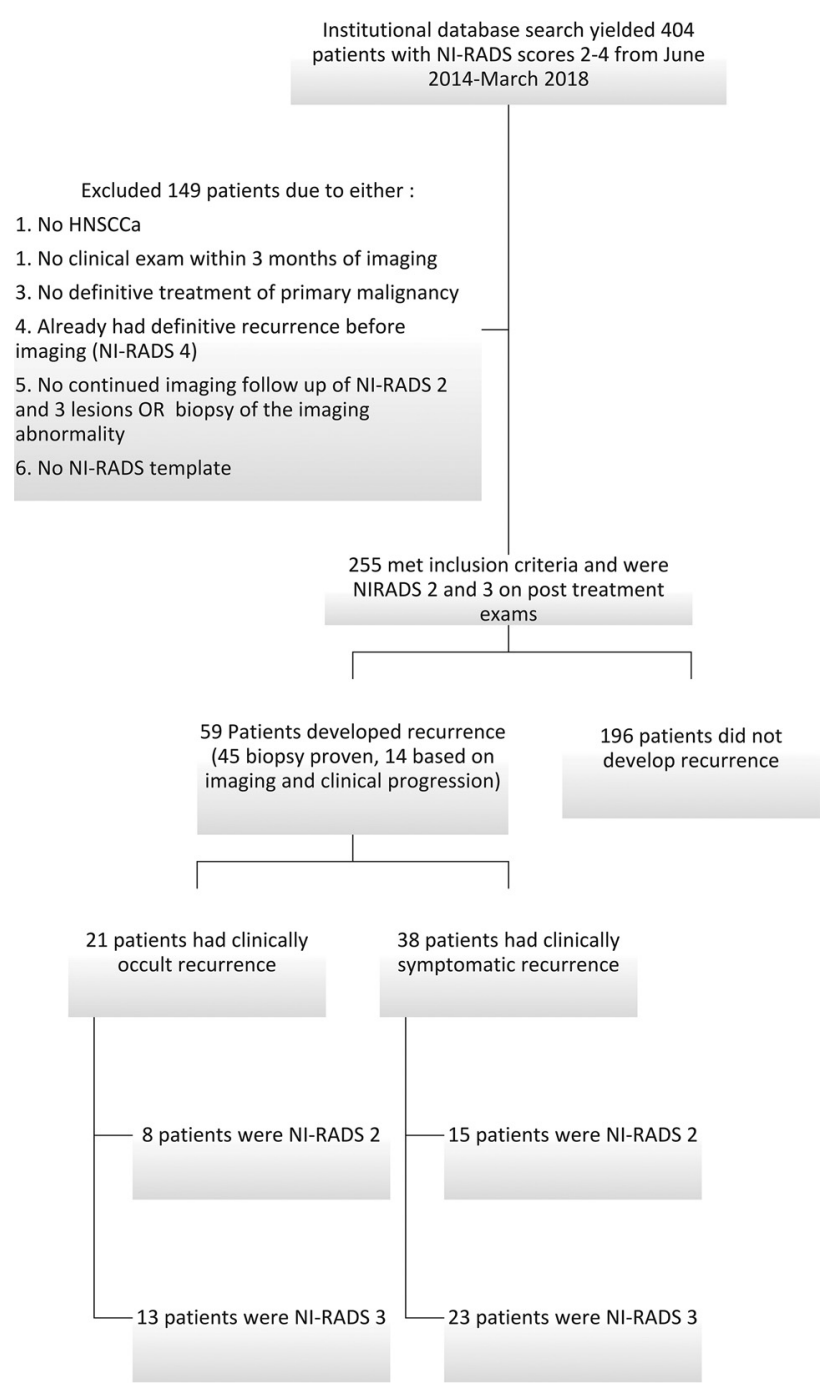

FIG 1. Flowchart demonstrating patient selection criteria and recurrences.

either did not have HNSCCa, did not strictly adhere to the NIRADS template, had no clinical follow-up within 3 months of imaging, had biopsy-proven recurrence before imaging (all 38 of NI-RADS 4 cases), or did not have follow-up of NI-RADS 2 or 3 lesions or biopsy of the imaging abnormality. A total of 255 cases with NI-RADS scores of $2(n=197)$ or $3(n=58)$ met the inclusion criteria (Fig 1). The 38 patients with NI-RADS 4 had either biopsy-proven locoregional $(n=33)$ recurrence or distant recurrence $(n=5)$ before obtaining a NI-RADS 4 on imaging. Therefore, these patients were excluded. Most of these patients were undergoing imaging to assess a response to chemotherapy in the setting of unsalvageable recurrence or distant metastasis or were on a clinical trial.

A total of 59 patients $(n=23$, NI-RADS $2 ; n=36$, NI-RADS 3) (23\%) had disease recurrence ( 45 biopsy-proven, 14 based on imaging progression on follow-up examination and clinical progression), with 17 recurrences diagnosed at $<6$ months, 16 recurrences between 6 and 12 months, 6 recurrences between 12 and 18 months, and 20 after 18 months. The biopsy-proven recurrences $(n=45)$ included 28 NI-RADS 3 and 17 NI-RADS 


\begin{tabular}{|c|c|c|c|c|c|c|c|c|}
\hline NI-RADS Score & $\begin{array}{l}\text { No. of } \\
\text { Cases }\end{array}$ & Sex & $\begin{array}{c}\text { Mean } \\
\text { Age (yr) }\end{array}$ & $\begin{array}{l}\text { Smoking } \\
\text { History }\end{array}$ & $\begin{array}{c}\text { Subsite of } \\
\text { Primary Tumor }\end{array}$ & T-Stage & HPV Status & Recurrences \\
\hline NI-RADS 2 & 197 & $\begin{array}{c}62 \mathrm{~F} \\
135 \mathrm{M}\end{array}$ & 64 & $75.6 \%$ (149) & $\begin{array}{l}\text { Oral cavity (73) } \\
\text { Oropharynx (48) } \\
\text { Hypopharynx (18) } \\
\text { Larynx (49) } \\
\text { Nasopharynx (7) } \\
\text { Unknown (2) }\end{array}$ & $\begin{array}{l}\text { T1 (35) } \\
\text { T2 (35) } \\
\text { T3 (32) } \\
\text { T4 (10) } \\
\text { T4a (56) } \\
\text { T4b (9) } \\
\text { Unknown (20) }\end{array}$ & $\begin{array}{l}32(+) \\
9(-) \\
2 \text { Unknown }\end{array}$ & $\begin{array}{l}\text { Total: } 23 \text { (8 clinically } \\
\text { occult recurrences) } \\
17 \text { Biopsy-proved } \\
6 \text { Imaging and } \\
\text { clinical progression }\end{array}$ \\
\hline NI-RADS 3 & 58 & $\begin{array}{l}17 \mathrm{~F} \\
41 \mathrm{M}\end{array}$ & 63 & $75.9 \%(44)$ & $\begin{array}{l}\text { Oral cavity (28) } \\
\text { Oropharynx (17) } \\
\text { Hypopharynx (4) } \\
\text { Larynx (8) } \\
\text { Nasopharynx (1) }\end{array}$ & $\begin{array}{l}\text { T1 (4) } \\
\text { T2 (14) } \\
\text { T3 (5) } \\
\text { T4 (8) } \\
\text { T4a (17) } \\
\text { T4b (6) } \\
\text { Unknown (4) }\end{array}$ & $\begin{array}{l}0(+) \\
1(-) \\
1 \text { Unknown }\end{array}$ & $\begin{array}{l}\text { Total: } 36 \text { (13 clinically } \\
\text { occult recurrences) } \\
28 \text { Biopsy-proved } \\
8 \text { Imaging and } \\
\text { clinical progression }\end{array}$ \\
\hline NI-RADS 4 & 38 & $\begin{array}{l}10 \mathrm{~F} \\
28 \mathrm{M}\end{array}$ & 63.4 & $71.1 \%$ (27) & $\begin{array}{l}\text { Oral cavity (8) } \\
\text { Oropharynx (16) } \\
\text { Hypopharynx (2) } \\
\text { Larynx (11) } \\
\text { Nasopharynx (1) }\end{array}$ & $\begin{array}{l}\text { T1 (4) } \\
\text { T2 (7) } \\
\text { T3 (6) } \\
\text { T4 (1) } \\
\text { T4a (15) } \\
\text { T4b (1) } \\
\text { Unknown (4) }\end{array}$ & $\begin{array}{l}8(+) \\
4(-) \\
1 \text { Unknown }\end{array}$ & $\begin{array}{l}\text { All patients excluded } \\
33 \text { Biopsy-proved } \\
\text { recurrence } \\
5 \text { Imaging and clinical } \\
\text { progression }\end{array}$ \\
\hline $\begin{array}{c}\text { Total (276 CECT, } \\
17 \text { PET/CECT) }\end{array}$ & 293 & $\begin{array}{c}89 \mathrm{~F} \\
204 \mathrm{M}\end{array}$ & 63.5 & $75.1 \%(220)$ & $\begin{array}{l}\text { Oral cavity (109) } \\
\text { Oropharynx (81) } \\
\text { Hypopharynx (24) } \\
\text { Larynx (68) } \\
\text { Nasopharynx (9) } \\
\text { Unknown (2) }\end{array}$ & $\begin{array}{l}\text { T1 (43) } \\
\text { T2 (56) } \\
\text { T3 (43) } \\
\text { T4 (19) } \\
\text { T4a (88) } \\
\text { T4b (16) } \\
\text { Unknown (28) }\end{array}$ & $\begin{array}{l}40(+) \\
14(-) \\
4 \text { Unknown }\end{array}$ & $\begin{array}{l}59 \text { NI-RADS } 2 \text { and } 3 \\
38 \text { NI-RADS } 4 \\
\text { (excluded) }\end{array}$ \\
\hline
\end{tabular}

2 recurrences. The recurrences based on imaging and clinical progression $(n=14)$ included 8 NI-RADS 3 and 6 NI-RADS 2. Twenty-one (36\%; eight NI-RADS 2 and 13 NI-RADS 3) patients with recurrence did not demonstrate clinical signs or symptoms at the time of recurrence diagnosis. Although our inclusion criteria required a clinical examination within 3 months of the index imaging examination, many were examined on the same day, with a mean time between clinical examination and the time of index imaging of only 11.3 days (range, 0-88 days). The Table and On-line Table summarize the demographics, T-staging, human papillomavirus (HPV) status, smoking history, and treatment of our study sample.

Of all clinically occult recurrences, $38 \%$ had a NI-RADS score of 2 and $62 \%$ had a score of 3 . Seventeen of these recurrences occurred at the primary site (14 submucosal and 3 mucosal), and 7 occurred within the lymph nodes. Time to recurrence was defined as the number of months from treatment to biopsyproven recurrence $(n=45)$ or definitive clinical and imaging progression $(n=14)$. The median time to clinically occult recurrence was $16.5 \pm 5.9$ months for NI-RADS 2 (range, 6.3-58.2 months, with outliers excluded) and $9.7 \pm 10.9$ months for patients with NI-RADS 3 (range, 3-107.9 months, with outliers excluded) (Fig 2). The combined median time to clinically occult recurrence was 11.4 months. Of all patients with clinically occult recurrence, 6 (29\%) patients underwent surgical salvage, $10(47 \%)$ were treated with re-irradiation and chemotherapy, 2 (10\%) patients opted for hospice care and were not candidates for salvage surgery, and $3(14 \%)$ patients sought salvage treatment at outside institutions. Examples of patients with clinically occult recurrences are shown in Figs 3 and 4.

\section{DISCUSSION}

HNSCCa is an aggressive malignancy with only about $50 \%$ of patients receiving an initial cure. Depending on the subsite of tumor and treatment, studies have demonstrated a wide range of recurrence from $22 \%$ to $86 \% .{ }^{14-16}$ We report a $23 \%$ recurrence rate (59 patients of 255) in our heterogeneous cohort.

Early identification of recurrence is critical for optimizing salvage therapy in attempts to improve survival and functional outcomes. The data regarding the exact relationship between early identification of recurrence and outcome are limited, especially the role of imaging to detect recurrences earlier than the clinical examination. To our knowledge, there are no data regarding the frequency of imaging-detected recurrences in asymptomatic patients, how much earlier imaging can detect recurrences, or the effect on outcome. This study is directed at answering the first question regarding the frequency of imaging-detected recurrence in asymptomatic patients. Although imaging surveillance in patients with HNSCCa is a common clinical practice, the technique, exact timing, and duration remains variable and inconsistent due to lack of data and guidelines. The NCCN only recommends surveillance imaging within the first 6 months, with no concrete recommendation beyond this period, despite many patients with head and neck carcinoma having a smoking history and recurrences often being submucosal. Previous studies have shown that the highest risk of recurrence occurs in the first 2 years after treatment. ${ }^{1,17,18}$ At our institution, asymptomatic patients follow the imaging surveillance recommendations outlined in the American College of Radiology NI-RADS White Paper, namely PET/CECT at 3 months followed by CECT at 6-month intervals $\times 2$ and then CECT of the neck at 1 -year 
Time to Recurrence in Patients Without Clinical Evidence of Disease

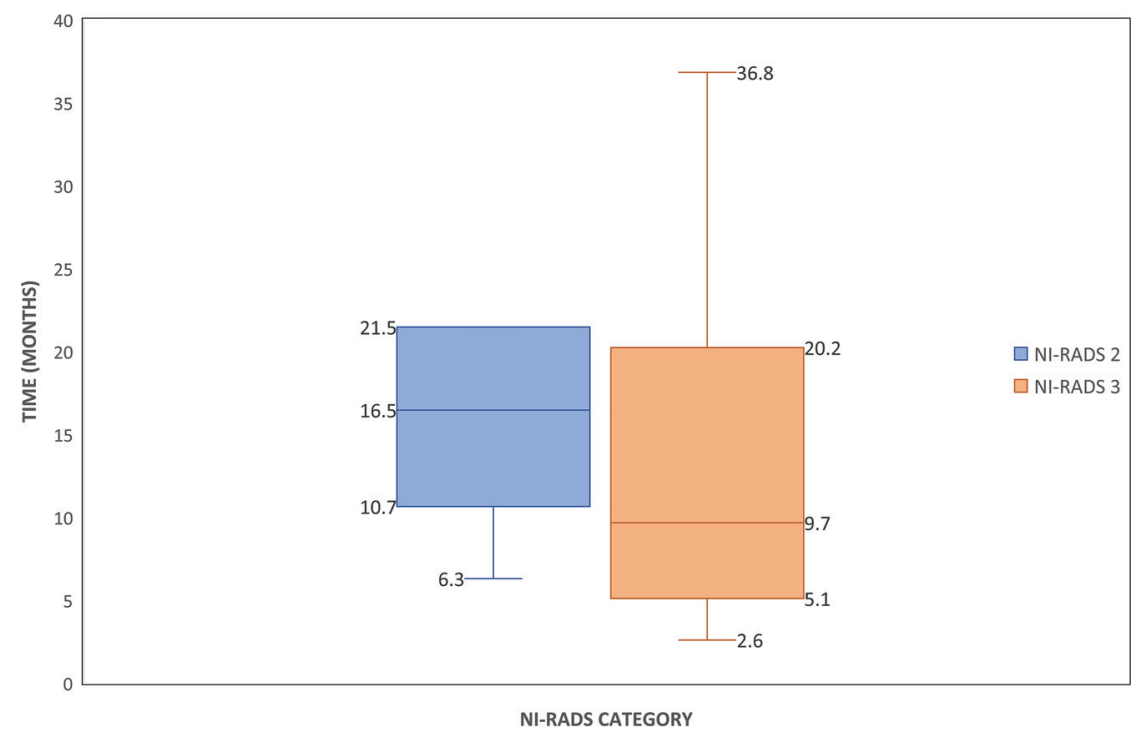

FIG 2. The median time to recurrence was $16.5 \pm 5.9$ months for NI-RADS 2 (range, 6.3-58.2 months, with outliers excluded) and $9.7 \pm 10.9$ months for NI-RADS 3 (range, 3-107.9 months, with outliers excluded). There are 2 outliers (not shown in figure) with values of 58.2 months for NIRADS-2 and 107.9 months for NI-RADS 3.

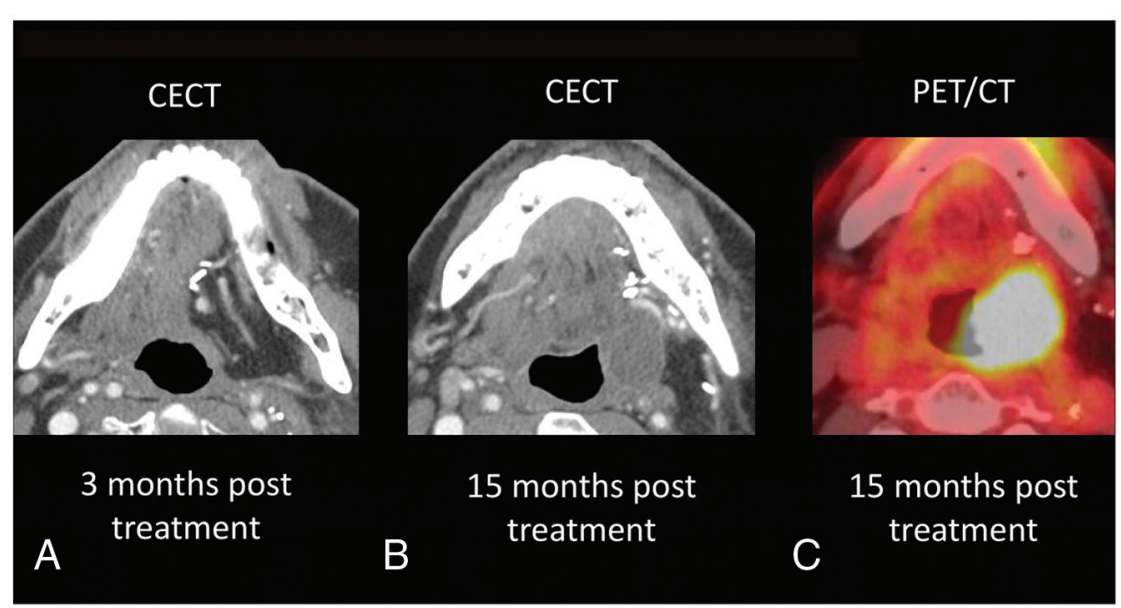

FIG 3. A, A 53-year-old man with a history of T2NOMO left-tongue squamous cell carcinoma status post left hemiglossectomy and flap reconstruction. Surveillance imaging demonstrates a new hypoattenuating mass within the left floor of the mouth along the flap margin (B), with corresponding hypermetabolism on PET/CT (C). On clinical examination, no oropharyngeal narrowing, bulge, or ulcerations were detected. The patient did not have worrisome clinical symptoms suspicious for clinical recurrence. This new mass was clinically occult and biopsy-proved recurrent disease. Recurrence was detected 15 months posttreatment.

intervals for 2 years in most patients. MR imaging surveillance is used for most sinonasal tumors, skull base tumors, salivary neoplasms, and any tumors with intracranial or intraorbital extension. Because our institution has a robust standard imaging algorithm for almost all advanced-stage HNSCCas using CECT and PET/CECT, we elected to study this cohort.

Imaging detected more than one-third of cases before clinical examination: Approximately $36 \%$ of patients (21 of 59 recurrences) with proven recurrence had no clinical evidence of recurrent disease, and the recurrence was detected by imaging alone (8 scored NI-RADS 2 and 13 scored NI-RADS 3). Our study demonstrates that imaging detected recurrence earlier than clinical examination in more than onethird of patients. There has been a growing number of studies assessing the value of $\left[{ }^{18} \mathrm{~F}\right]$ FDG-PET/CT in posttreatment surveillance imaging, with many authors arguing that routine follow-up is critical, $7,9,10,12,19$ and some have even gone further to suggest site-specific recommendations. $^{20,21}$ One can hypothesize that imaging surveillance may be especially important in patients with asymptomatic locoregional recurrences that may be salvageable, improving disease-free survival. ${ }^{22}$

Imaging detected subclinical recurrences beyond 6 months in most patients: Our standardized surveillance algorithm enabled detection of recurrences beyond 6 months and earlier than clinical examination/ symptoms in $81 \%$ of asymptomatic patients (17 of 21 ), though we do not have data regarding the effect on outcome. This data, revealing a noteworthy number of clinically occult recurrences with a large percentage occurring beyond 6 months, would argue in favor of a more robust surveillance algorithm beyond the posttreatment baseline examination recommended by the NCCN guidelines. The exact frequency and duration can likely be tailored on the basis of the initial stage, HPV status, smoking history, and so forth and is an important area for future study. A study by Abidi et $\mathrm{al}^{23}$ demonstrated a lower frequency of subclinical recurrences with FDG-PET/CT in $8 \%$ of surveyed patients between 8 and 36 months. Some studies have also demonstrated improved survival outcomes with earlier detection of recurrences beyond the first 6 months. ${ }^{5,24,25}$ For example, Wong et $\mathrm{al}^{24}$ demonstrated that the overall 5 -year tumor-free survival following surgical salvage was $26 \%$, whereas those patients with late detection or those whose disease was not considered salvageable either via surgery, radiation therapy, or chemotherapy only achieved a mean survival of 7 months. Imaging is critical for identifying early disease recurrence, which often occurs beyond 6 months and can be seen in patients without worrisome clinical and physical examination findings. 


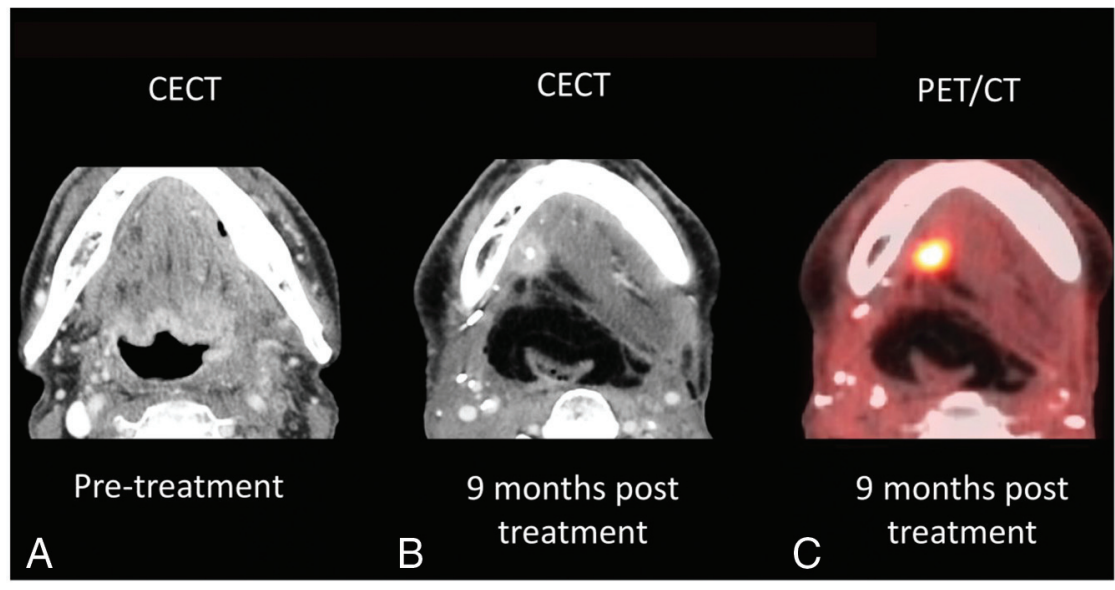

FIG 4. A 62-year-old-man with pT4aN2b HPV-negative squamous cell carcinoma of the left base of the tongue with extension into the floor of mouth and inferiorly to the hypopharynx (partially visualized in A). Surgically, the patient underwent total glossectomy and laryngectomy with a left anterolateral thigh flap reconstruction of the pharynx and base of the tongue. $B$, An enhancing $1.4-\mathrm{cm}$ nodule with central cystic change in the right floor of the mouth along the margin of the flap. On clinical examination, no mucosal masses, bulges, or suspicious ulcerations were detected. The patient did not have worrisome clinical symptoms to suspect clinical recurrence. This new mass along the flap margin demonstrated marked FDG avidity on PET/CT (C) and was biopsyproved recurrence. Recurrence was detected 9 months posttreatment.

\section{Treatment Options for Recurrences}

The concept that earlier detection of recurrence in asymptomatic patients yields a survival benefit is controversial and needs further study; some authors have shown a survival benefit, ${ }^{20,26}$ whereas others have not. ${ }^{27-31}$ Although this study was not designed or powered to assess outcome or survival, we retrospectively investigated treatment options used for our recurrences. In our study, 6 patients (29\%) with clinically occult recurrence underwent salvage surgery ( $n=4$, NI-RADS 2 ; and $n=2$. NI-RADS 3 ) with curative intent. One (5\%) patient with NI-RADS 3 underwent chemoradiation with curative intent, while the remaining patients with clinically occult recurrence received palliative care (with 1 patient forgoing surgical salvage with curative intent). In patients who had symptomatic recurrence, $24 \%(n=9)$ underwent salvage surgery with curative intent.

When we compared the 2 scenarios, 29\% $(n=6)$ of our patients with clinically occult recurrence were able to undergo successful surgical salvage compared with $24 \%(n=9)$ of patients with symptomatic recurrence. For patients with locoregional recurrence in addition to distant metastases, treatment was primarily palliative. A total of 3 patients with symptomatic recurrence declined further treatment: Two decided on hospice care, and one was unable to undergo treatment due to a lifethreatening infection. Ten patients were treated with re-irradiation and chemotherapy in both groups. Although this study was not designed to determine survival benefit or the effect on treatment options of early imaging detection, the authors believe that they are very important questions and would be the next step for optimizing surveillance.

\section{Mucosal Recurrence}

In addition to identifying asymptomatic submucosal recurrences, our study also identified 2 asymptomatic mucosal recurrences (albeit, one with nodal involvement as well). One patient was examined at an outside facility 30 days prior to imaging without an endoscopy report, and the other demonstrated recurrence in the oropharynx in addition to node involvement. In these 2 patients, the clinical examination was an average of 14 days before the imaging examination. Just as posttreatment imaging is complicated in the patient with posttreatment head and neck cancer, the mucosal examination can be difficult, especially in the radiated larynx. Although typically the clinical exam is the workhorse for detecting mucosal recurrences and imaging for submucosal recurrences, perhaps unexpectedly, imaging can also play a role in detecting asymptomatic mucosal recurrences. The complex post radiated mucosa can make the clinical exam difficult, and imaging can help to direct the clinical exam for added

sensitivity in some cases.

\section{Limitations}

The primary limitations of our study were the retrospective design, a heterogeneous population with a mixture of stages and subsites, and sample size. The primary aim of our article was to show that a standardized imaging surveillance algorithm resulted in earlier detection, with the logical next question being whether earlier detection would result in different treatment options for the patient and/or survival benefits. Our study was not designed to answer these questions, predominately due to its retrospective nature without a control group and a heterogeneous cohort with mixed subsites, stages, HPV status, and risk factors such as smoking history. At our institution, all patients undergo standard surveillance imaging; thus, we do not have access to a cohort of asymptomatic patients followed by clinical examination alone for comparison. Intuitively, those patients for whom early imaging detection of disease recurrence offers more options for surgical salvage should have better prognoses than those whose recurrences are detected after involving vital structures like the carotid artery, skull base, or prevertebral musculature. We believe this is a critical question but very difficult to design a prospective study for because most HNSCCas do undergo some form of surveillance imaging in asymptomatic patients, albeit nonstandardized. This study also followed patients only to determine the presence of disease either by biopsy or progression or the absence of disease by resolution of abnormal findings on imaging on subsequent follow-up. A long-term follow-up of this cohort would be needed to assess whether earlier radiographic detection translates into better outcomes and improved survival. Our study was also underpowered for subset analysis to extrapolate whether certain patient groups (eg, higher tumor stage, smoking history, HPV status, subsite, and so forth) should warrant more frequent 
surveillance. We believe that this is an important next question for future larger, multi-institutional studies.

\section{CONCLUSIONS}

Imaging surveillance beyond the first posttreatment baseline study was critical for earlier detection of recurrent disease. More than one-third of all imaging-detected recurrences were seen in patients with no clinical evidence of disease. Most of these recurrences occurred beyond 6 months from the end of treatment. These findings underscore the importance of extendimg the current NCCN guidelines of imaging surveillance beyond the first 6 months of treatment. Further study is warranted to determine which patients would benefit most from asymptomatic imaging surveillance.

Disclosures: Mihir R. Patel—UNRELATED: Travel/Accommodations/Meeting Expenses Unrelated to Activities Listed: Intuitive Surgical.

\section{REFERENCES}

1. Beswick DM, Gooding WE, Johnson JT, et al. Temporal patterns of head and neck squamous cell carcinoma recurrence with positron-emission tomography/computed tomography monitoring. Laryngoscope 2012;122:1512-17 CrossRef Medline

2. Isles MG, McConkey C, Mehanna HM. A systematic review and meta-analysis of the role of positron emission tomography in the follow up of head and neck squamous cell carcinoma following radiotherapy or chemoradiotherapy. Clin Otolaryngol 2008;33:21022 CrossRef Medline

3. Krieger DA, Hudgins PA, Nayak GK, et al. Initial performance of NI-RADS to predict residual or recurrent head and neck squamous cell carcinoma. AJNR Am J Neuroradiol 2017;38:1193-99 CrossRef Medline

4. Aiken AH, Farley A, Baugnon KL, et al. Implementation of a novel surveillance template for head and neck cancer: Neck Imaging Reporting and Data System (NI-RADS). J Am Coll Radiol 2016;13:743-46.e1 CrossRef Medline

5. Aiken AH, Rath TJ, Anzai Y, et al. ACR Neck Imaging Reporting and Data Systems (NI-RADS): A White Paper of the ACR NI-RADS Committee. J Am Coll Radiol 2018;15:1097-1108 CrossRef Medline

6. Hsu D, Chokshi FH, Hudgins PA, et al. Predictive value of first posttreatment imaging using standardized reporting in head and neck cancer. Otolaryngol Head Neck Surg 2019;161:978-85 CrossRef Medline

7. McDermott M, Hughes $M$, Rath $T$, et al. Negative predictive value of surveillance PET/CT in head and neck squamous cell cancer. AJNR Am J Neuroradiol 2013;34:1632-36 CrossRef Medline

8. Wangaryattawanich $\mathrm{P}$, Branstetter BF, Hughes M, et al. Negative predictive value of NI-RADS category 2 in the first posttreatment FDG-PET/CT in head and neck squamous cell carcinoma. AJNR Am J Neuroradiol 2018;39:1884-88 CrossRef Medline

9. Koshkareva Y, Branstetter BF, Gaughan JP, et al. Predictive accuracy of first post-treatment PET/CT in HPV-related oropharyngeal squamous cell carcinoma. Laryngoscope 2014;124:1843-47 CrossRef Medline

10. Leung AS, Rath TJ, Hughes MA, et al. Optimal timing of first posttreatment FDG PET/CT in head and neck squamous cell carcinoma. Head Neck 2016;38(Suppl 1):E853-58 CrossRef Medline

11. Ong SC, Schöder H, Lee NY, et al. Clinical utility of 18F-FDG PET/CT in assessing the neck after concurrent chemoradiotherapy for locoregional advanced head and neck cancer. J Nucl Med 2008;49:532-40 CrossRef Medline

12. Gilbert MR, Branstetter BF, Kim S. Utility of positron-emission tomography/computed tomography imaging in the management of the neck in recurrent laryngeal cancer. Laryngoscope 2012;122:82125 CrossRef Medline
13. Ang KK, Trotti A, Brown BW, et al. Randomized trial addressing risk features and time factors of surgery plus radiotherapy in advanced head-and-neck cancer. Int J Radiat Oncol Biol Phys 2001;51:571-78 CrossRef

14. Vikram B, Strong EW, Shah JP, et al. Failure at the primary site following multimodality treatment in advanced head and neck cancer. Head Neck Surg 1984;6:720-23 CrossRef Medline

15. Mizrachi A, Migliacci JC, Montero $\mathrm{PH}$, et al. Neck recurrence in clinically node-negative oral cancer: 27 -year experience at a single institution. Oral Oncol 2018;78: 94-101 CrossRef Medline

16. Manikantan K, Khode S, Dwivedi RC, et al. Making sense of posttreatment surveillance in head and neck cancer: when and what of follow-up. Cancer Treat Rev 2009;35:744-53 CrossRef Medline

17. Schwartz DL, Barker J, Chansky K, et al. Postradiotherapy surveillance practice for head and neck squamous cell carcinoma: too much for too little? Head Neck 2003;25:990-99 CrossRef Medline

18. Wensing BM, Merkx MAW, Krabbe PFM, et al. Oral squamous cell carcinoma and a clinically negative neck: the value of follow-up. Head Neck 2011;33:1400-05 CrossRef Medline

19. Ghosh-Laskar S, Mummudi N, Rangarajan V, et al. Prognostic value of response assessment fluorodeoxyglucose positron emission tomography-computed tomography scan in radically treated squamous cell carcinoma of head and neck: long-term results of a prospective study. J Cancer Res Ther 2019;15:596-603 CrossRef Medline

20. de Visscher AV, Manni JJ. Routine long-term follow-up in patients treated with curative intent for squamous cell carcinoma of the larynx, pharynx, and oral cavity: does it make sense? Arch Otolaryngol Head Neck Surg 1994;120:934-39 CrossRef Medline

21. Boysen M. Value of follow-up in patients treated for squamous cell carcinomas of the oral cavity and oropharynx. Recent Results Cancer Res 1994;134:205-14 CrossRef Medline

22. Janot F, de Raucourt D, Benhamou E, et al. Randomized trial of postoperative reirradiation combined with chemotherapy after salvage surgery compared with salvage surgery alone in head and neck carcinoma. J Clin Oncol 2008;26:5518-23 CrossRef Medline

23. Abidi S, Vasanawala M, Segall G. Is F18-FDG PET useful in surveillance of head and neck cancer. Journal of Nuclear Medicine 2019;60(Suppl 1):1258

24. Wong LY, Wei WI, Lam LK, et al. Salvage of recurrent head and neck squamous cell carcinoma after primary curative surgery. Head Neck 2003;25:953-59 CrossRef Medline

25. Lowe VJ, Boyd JH, Dunphy FR, et al. Surveillance for recurrent head and neck cancer using positron emission tomography. J Clin Oncol 2000;18:651-58 CrossRef Medline

26. Ritoe SC, de Vegt F, Scheike IM, et al. Effect of routine follow-up after treatment for laryngeal cancer on life expectancy and mortality: results of a Markov model analysis. Cancer 2007;109:239-47 CrossRef Medline

27. Lester SE, Wight RG. 'When will I see you again?' Using local recurrence data to develop a regimen for routine surveillance in post-treatment head and neck cancer patients. Clin Otolaryngol 2009;34:546-51 CrossRef Medline

28. Boysen M, Lövdal O, Tausjö J, et al. The value of follow-up in patients treated for squamous cell carcinoma of the head and neck. Eur J Cancer 1992;28:426-30 CrossRef Medline

29. Francis DO, Yueh B, Weymuller EA, et al. Impact of surveillance on survival after laryngeal cancer in the Medicare population. Laryngoscope 2009;119:2337-44 CrossRef Medline

30. Saussez S, Dekeyser C, Thill MP, et al. Importance of clinical and radiological follow-up in head and neck cancers. B-ENT 2007;3:17984 Medline

31. De Felice F, Musio D, Tombolini V. Follow-up in head and neck cancer: a management dilemma. Advances in Otolaryngology 2015; 2015:1-4 CrossRef 